\title{
The spatiotemporal responses of Populus euphratica to global warming in Chinese oases between 1960 and 2015
}

\author{
ZHANG Wenxia, "LIU Puxing, FENG Qingrong, WANG Tianguang, \\ WANG Tianqiang \\ College of Geography and Environmental Science, Northwest Normal University, Lanzhou 730070, China
}

\begin{abstract}
Daily average temperature data from 48 meteorological stations in Chinese oases that are within the distribution area of Populus euphratica were analyzed to determine the spatiotemporal responses of this tree to climate change. Specifically, the start and end date as well as the number of days that comprised the growing season were analyzed with a multi-year trend line and using the Mann-Kendall mutation test, inverse distance weighted interpolation (IDW) in the software ArcGIS, a Morlet wavelet power spectrum, and correlation analysis. The results of this study show that, over the last 56 years, the start date of the $P$. euphratica growing season has advanced, while the end date has been postponed, and the number of days that comprise the growing season have gradually increased. The changing trend rates recovered in this analysis for these three time slices are $-1.34 \mathrm{~d} / 10 \mathrm{a}, 1.33 \mathrm{~d} / 10 \mathrm{a}$, and $2.66 \mathrm{~d} / 10 \mathrm{a}(\alpha \geqslant 0.001)$, respectively. Data show that while spatial disparity is extremely significant, it is nevertheless the case that along a southwest-to-northeast transect of Chinese oases, the later the start date of the $P$. euphratica season, the sooner the end data and the shorter the growing season. Mutations points in start and end date, as well as for the growing season overall were observed in 2001,1989, and 1996, respectively, and the data presented in this paper show that, in particular, the date of this end of this period is most sensitive to climate warming. Growing season cycles for $P$. euphratica are between 3.56 years and 7.14 years, consistent with the periodicity of EI Niño events, while a start date cycle between 3.56 years and 4.28 years is consistent with atmospheric circulation cyclicity. The causal analysis presented in this paper shows that the Asian polar vortex area index (APVAI), the Qinghai-Tibet Plateau index (TPI), the westerly circulation index (WCI), and carbon dioxide emissions (CDE) are the main factors influencing spatiotemporal changes in the growth of $P$. euphratica, the effect of latitude during the growing season is more significant than altitude, and the start date of the growing season is more significantly influenced by these factors than end date. In addition, data show that the start date, end date, and length of the growing season are all significantly correlated with their average corresponding monthly temperature (corre-
\end{abstract}

Received: 2017-09-17 Accepted: 2017-10-30

Foundation: National Natural Science Foundation of China, No.40961035, No.41461012; The Science and Technology Project of Gansu Province, No.0803RJZA094

Author: Zhang Wenxia (1987-), MS Candidate, specialized in arid area environment and the construction of oasis. E-mail: zwxjiayou@sina.com

"Corresponding author: Liu Puxing (1964-), PhD and Professor, E-mail: liupx2016@163.com 
lation coefficients are $-0.875,0.770$, and $0.897 ; \alpha \geqslant 0.001)$. Thus, if the average temperature in March increases by $1{ }^{\circ} \mathrm{C}$, the start date of the growing season will advance by 2.21 days, while if the average temperature in October increases by the same margin then the seasonal end date will be delayed by 2.76 days. Similarly, if the average temperature between March and October increases by $1^{\circ} \mathrm{C}$, the growing season will be extended by 7.78 days. The results of this study corroborate the fact that changes in the $P$. euphratica growing are sensitive to regional warming and are thus of considerable theoretical significance to our understanding of the responses of Chinese vegetation to climate change as well as to ecological restoration.

Keywords: Populus euphratica; annual growing season; global warming; responses to climate change; Chinese oases

\section{Introduction}

Global warming has been occurring for almost a century. The Fifth Assessment Report of the Intergovernmental Panel on Climate Change noted that global average surface temperature increased by $0.85^{\circ} \mathrm{C}$ between 1880 and 2012, and it is also the case that warming trends within China are consistent with those seen across the rest of the world (Qin et al., 2014). Vegetation is the most important component of terrestrial ecosystems, and so the responses of plant phenology to regional climate change are both sensitive and simple to observe (Chmielewski and Rötzer, 2001). This means that research on the responses of plant phenology to global climate change have become a hot topic both within China and internationally (Fang and Yu, 2002); a number of previous studies have concluded that global warming has led to corresponding changes in the start and end dates of growth as well as the length of this period in plants (Pei et al., 2009; Zhang, 1995). Research outside China began much earlier; organized phenological observations in Europe, for example, were first initiated in the middle of the 18th century, and a number of devoted observation networks were subsequently established, including the European Penology Network and the International Phenological Gardens (IPG) (Pei et al., 2009). Based on analysis of IPG data from between 1960 and 1990, Menzel et al. (1999) noted that spring events have advanced by 6 days and autumn events have been delayed by 4.8 days likely because of rising European temperatures. Similarly, spring events in North American lilac (Schwartz and Reiter, 2000) and Belgian temperate woody plants (Fu et al., 2012) have also advanced markedly, while a $1{ }^{\circ} \mathrm{C}$ increasing in early spring temperature has been shown to have advanced the start date of the Betula pubescens and Prunus avium growing season in Europe by 8 days (Chmielewski and Rötzer, 2001). More than $70 \%$ of the Northern Hemisphere areas surveyed exhibit a trend towards the postponement of the growing season end date (mean rate: $0.18 \pm 0.38 \mathrm{~d} / \mathrm{a}$; Liu et al., 2016), and phenological responses to climate change are also evident in the start of flowering, leaf-unfolding, and veraison periods. Previously presented data show that the start of flowering has markedly advanced in a number of central European plants (i.e., snowdrop, forsythia, sweet cherry, and apple) (Roetzer et al., 2000), as well as in 11 UK species (Sparks et al., 2000), P. tremuloides in Canada (Beaubien and Freeland, 2000), citrus in Kerman and Shiraz in Iran (Fitchett et al., 2014), and in several Hungarian taxa (i.e., Convallaria majalis, Taraxacum officinale, Sambucus nigra, Tilia cordata) (Szabó et al., 2016). Similarly, the leaf-unfolding period in Swiss horse-chestnut trees (Defila and Clot, 2001) as well as in Lithuanian deciduous trees (Juknys et al., 2016), and the veraison of the Rkatsiteli in Georgia (Cola et al., 2016) have also all significantly advanced. The founder of 
modern Chinese phenology, Zhu, established a national observation network in 1963 to provide data for the study of changes (Ge et al., 2010), while Zhang (1995) pointed out that temperature was likely the most important meteorological factor affecting growth changes in woody plants. Zhang (1995) also noted that when annual mean temperature increases by $1^{\circ} \mathrm{C}$, spring events in Chinese woody plants advanced by between 3 and 4 days, while autumn events were delayed by the same time period. A number of scholars have studied phenological changes in Chinese temperate plants (Chen et al., 2015), herbs in Hebei Province (Gao et al., 2012), woody plants in the city of Guiyang (Bai et al., 2009), general species within the city of Zhengzhou (Liu et al., 2007), and the vegetation of the Minqin desert area (Chang et al., 2009) to demonstrate advances in spring growth events correlated with autumn postponements. Chen et al. (2007) also noted that the length of the multi-year average growing season is mainly influenced by variations in latitude and altitude.

The tree species Populus euphratica is known to occur in 20 countries across Asia, Africa, and Europe, and is one of the most widespread desert region taxa in Central Asia. The highest abundance and largest area of this species globally are found within Chinese oases, mainly in Xinjiang Province, the Qaidam Basin, the Alxa Plateau, the Hexi Corridor, and on the Hetao Plain (Wei, 1990; Wang et al., 1995; Liu and Zhang, 2011). This species has the advantage of being drought and salt tolerant, and is also wind resistant, fast growing, and highly adaptable (Wei, 1990). Indeed, as $P$. euphratica is 'a green guard' that maintains the ecological balance of arid area oases, changes in its growing season start and end date as well as the length of this period are clear indicators of the impact of climate change on Chinese oases. Previous studies to evaluate the growing season responses of $P$. euphratica to climate change were focused on Hexi Corridor (Liu and Zhang, 2011) and Ejina Banner oases (Zhao et al., 2012), as well as some other isolated regions; the vast oases in Xinjiang Province, on the Hetao Plain, and in the Qaidam Basin have so far not been studied, and so the patterns and drivers of spatiotemporal responses to climate change remain unknown. The aim of this study is therefore to evaluate the spatiotemporal characteristics of growing season changes in $P$. euphratica within these regions in order better understand the effects of climate change and to provide a theoretical basis for the restoration and reconstruction of the ecological environment in Chinese oases.

\section{Data and methods}

\subsection{Study area}

Chinese oases are mainly distributed within the desert and semi-desert regions of northwestern China, between $34^{\circ} 25^{\prime}-48^{\circ} 10^{\prime} \mathrm{N}$ and $73^{\circ} 40^{\prime}-109^{\circ} 08^{\prime} \mathrm{E}$, almost entirely in high mountains and on huge proluvium fans (Figure 1). These oases are mostly subject to a desert climate which consists of droughts and rare rainfall, are cold in the winter and hot in the summer, and experience large ranges of both annual and daily temperature. These oases are also heat-rich, a trend that increases generally from the east to the west; an accumulated temperature of $10^{\circ} \mathrm{C}$ or greater is seen as values increase from $2500^{\circ} \mathrm{C}$ in the Hexi Corridor to $4500^{\circ} \mathrm{C}$ within the Tarim Basin in Xinjiang Province, while the accumulated temperature of the Qaidam Basin is very low (mostly less than $1500^{\circ} \mathrm{C}$ ) as it is influenced by the vertical zone of the Tibetan Plateau. Mean annual rainfall in these regions tends to be less than 200 
$\mathrm{mm}$, while evaporation is very strong and sunshine levels are high, especially in the Hexi Corridor, the Tarim Basin, and western Inner Mongolia where annual solar radiation is above $586 \mathrm{~kJ} / \mathrm{cm}^{2}$ (Shen et al., 2001). The soils of these regions are also dominated by brown-to-gray- brown deserts and eolian sandy soils, while zonal vegetation is mostly characterized by desert and desert steppe.

\subsection{Data}

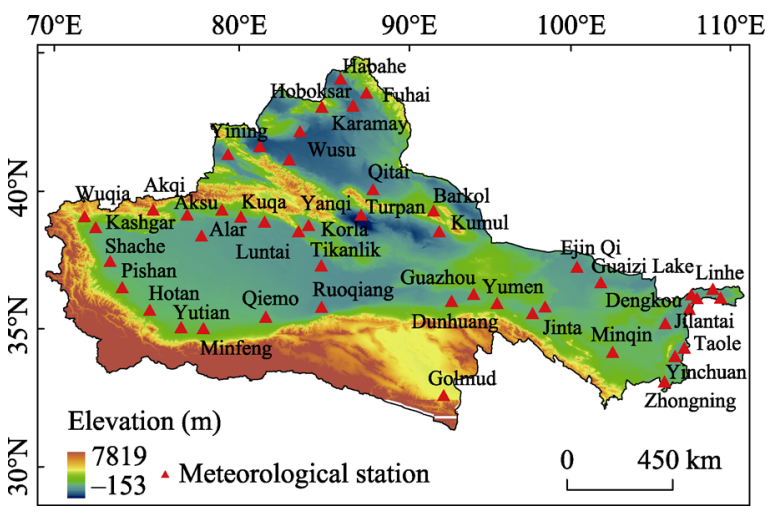

Figure 1 Distribution of meteorological stations in Chinese oases characterized by $P$. euphratica

A dataset of daily average temperatures was used in this study that encompasses the period between 1960 and 2015; these data were recorded at 48 meteorological stations that span the distributional range of $P$. euphratica, all downloaded from the China Meteorological Science Data-sharing Service System (http://www.cma.gov.cn/). In earlier work, Shen et al. (2001) divided Chinese oasis regions into six parts, northern and southern Xinjiang Province, the Hexi Corridor, the Hetao Plain, the Qaidam Basin, and the Alxa Plateau. Similarly, and on the basis of observations of 42 plants in the Minqin desert botanical garden, Chang et al. (2009) considered temperature to be the main factor influencing the phenology of oasis plants in arid areas; indeed, these workers suggested that increasing temperature was the main reason underlying changes in the phenology of plants within the Minqin desert area. Based on the standard division proposed by Wei (1990), the annual cycle of $P$. euphratica starts with an average daily temperature of $5^{\circ} \mathrm{C}$ or greater that remains stable throughout the spring, and ends at an average daily temperature at this level or less that remains stable throughout the autumn. We therefore confirmed the start date, the end date, and the growing season for $P$. euphratica, and applied an average weighting method to subregions when calculating these variables for Chinese oases. The circulation feature indexes used in this study were drawn from a set of 74 that comprise monthly values for the period between 1960 and 2015 issued by National Climate Center, including the cold air index (CA), the Tibetan Plateau index (TPI), the Asian polar vortex index (APVII, APVAI), and the westerly circulation index (WCI). In addition, annual mean carbon dioxide emissions (CDE) for the period between 1960 and 2011 were extracted from the World Bank database of world development indicators (http://data.worldbank.org.cn/). We analyzed trends and variations in the $P$. euphratica growing season applying a linear fitting method, and evaluated spatial variation and disparity via inverse distance weighted interpolation (IDW) in the software ArcGIS 9.3, and utilized the Mann-Kendall method for mutation tests as well as the Morlet wavelet power spectrum for periodic analysis. Finally, we discuss the factors that influence aspects of the $P$. euphratica growing season via correlation and regression analyses.

\section{Results}

\subsection{Temporal variations in the $P$. euphratica growing season}

(1) The start of growing season

The data presented in this paper show that over the past 56 years, variation in the start date of the $P$. euphratica growing season in Chinese oases has advanced significantly at a 
rate of $-1.34 \mathrm{~d} / 10 \mathrm{a}(\alpha \geqslant 0.001)$ (Figure $2 \mathrm{a})$, while the average start date (March $21 \mathrm{st}$ ) has advanced 7.5 days over the past 56 years. Interestingly, although the start date in each oasis was not the same, they had all advanced to varying degrees (Figure 3a1-3f1); amongst these, the start date in the southern Xinjiang oasis was the earliest, March 11th on average, while that in the Qaidam Basin oasis was the latest, April 8th on average. The most obvious advancing trend was seen in the Hetao Plain oasis, where the change rate is $-2.55 \mathrm{~d} / 10 \mathrm{a}$ ( $\alpha \geqslant$ 0.001 ); this start date has advanced 14 days over the last 56 years. The most unclear trend in advancing start date was seen in the southern Xinjiang oasis where the rate of change is $-0.95 \mathrm{~d} / 10$ a $(\alpha \geqslant 0.05)$; the start date in this oasis has advanced by just 5.4 days over the past 56 years.

In terms of inter-decadal variations (Table 1), the anomaly value of the start date was positive between the 1960s and the 1980s; this encapsulates a slight trend towards a delay during the 1970s, as well as a return to an advance during the 1980s. In the 1990s, however, this anomaly value reverted to negative; the observed change in this anomaly was the greatest between the 1990s and 2000s, reaching a maximum value of 3.8 days in the 2000 s. These data show that the most significant advancing trend was during the $2000 \mathrm{~s}$.
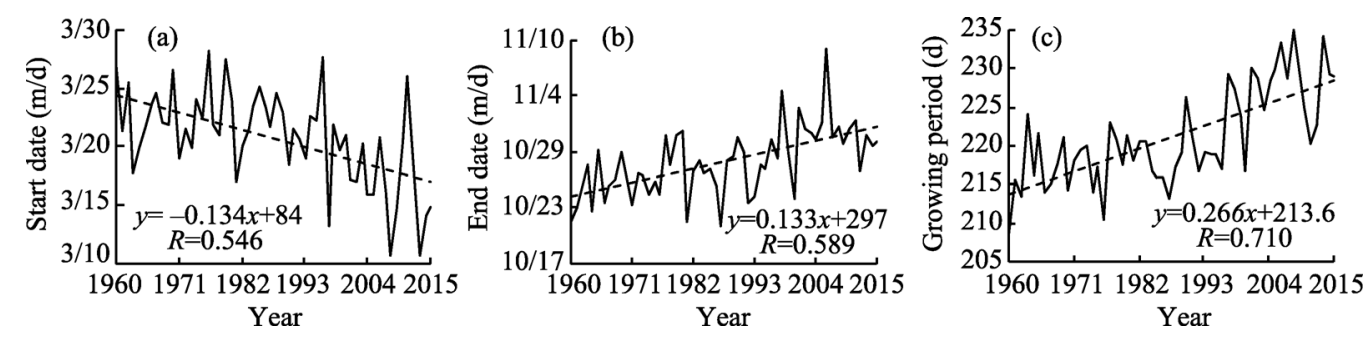

Figure 2 Trends in P. euphratica start date, end date, and growing season variation in Chinese oases

(2) The end of growing season

Data presented in this study reveal that, over the past 56 years, variation in the end date of the $P$. euphratica growing season in Chinese oases has tended to be delayed significantly at a rate of $1.33 \mathrm{~d} / 10 \mathrm{a}(\alpha \geqslant 0.001)$ (Figure $2 \mathrm{~b}$ ). Indeed, the average growing season end date has been delayed by 7.5 days over the past 56 years and now ends on October 28th on average. As with start dates, the recorded end date in each oasis is not the same and they have all been delayed by varying degrees (Figure 3 a2-3f2). Amongst these data, the end date for the P. euphratica growing season in the Qaidam Basin oasis was the earliest, ending on October 16 th on average, while that of the southern Xinjiang oasis was the latest, ending on November 1 st on average. The most obvious trend in postponement was seen in the northern Xinjiang oasis, where the rate of change is $1.83 \mathrm{~d} / 10 \mathrm{a}(\alpha \geqslant 0.001)$; over the past 56 years, the end date has been delayed by 10 days in this oasis, while the most ambiguous trend towards postponement was in the Hetao Plain oasis, a rate of $1.02 \mathrm{~d} / 10 \mathrm{a}(\alpha \geqslant 0.05)$. In this latter case, the end date of the growing season has been delayed by just 5.7 days over the past 56 years.

In terms of inter-decadal variations (Table 1), the anomaly value of the end date was negative between 1960 and the 1980s; the value of this anomaly ranged between -2.7 and 1.5 , while the end date exhibited a slight trend towards advancement during the 1980s. In contrast, the end date tended to be delayed during the 1990s; this value switched from negative to 
(a1) Nanjiang
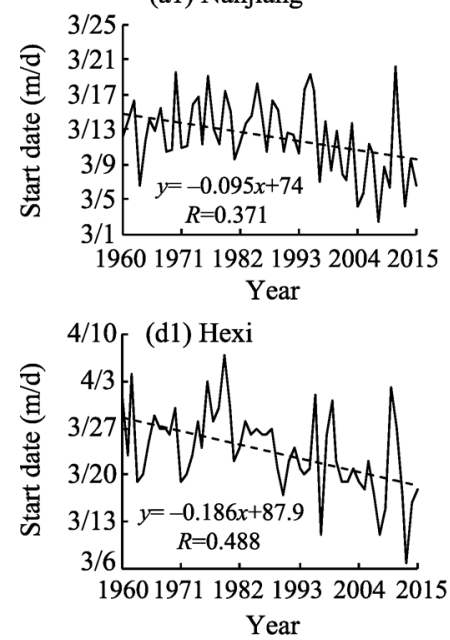

(a2) Nanjiang

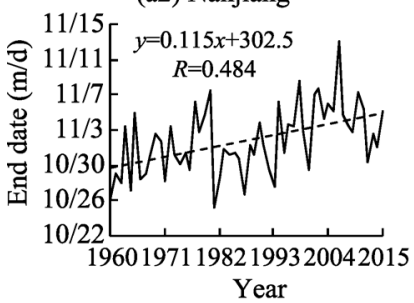

(d2) Hexi

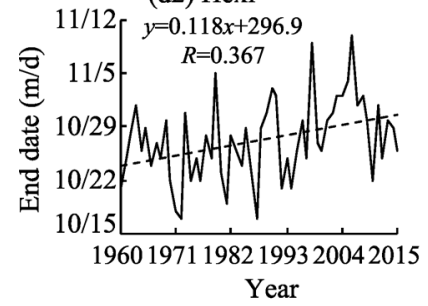

(a3) Nanjiang

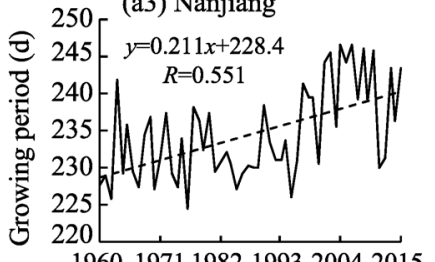

Year

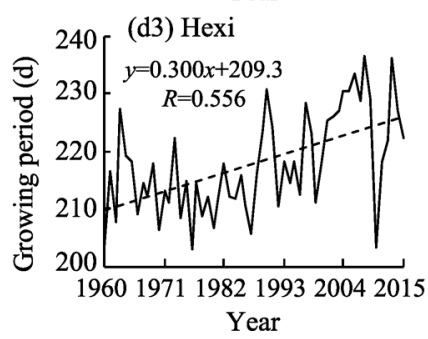

(b1) Beijiang
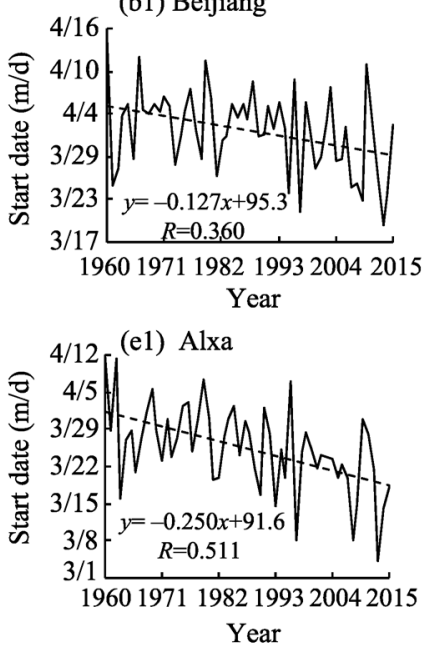

(b2) Beijiang

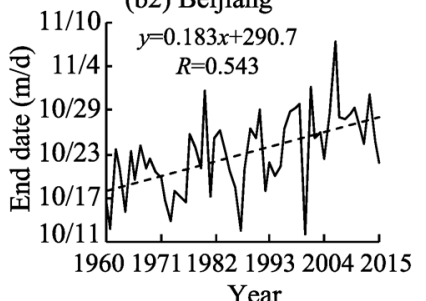

(e2) Alxa

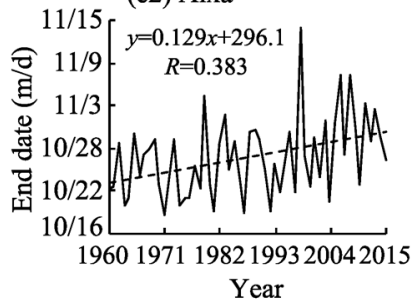

(b3) Beijiang

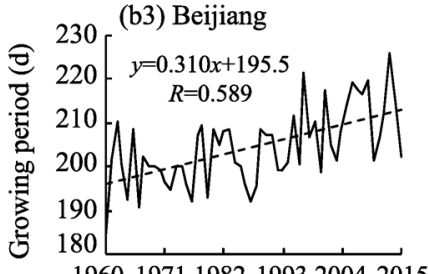

Year

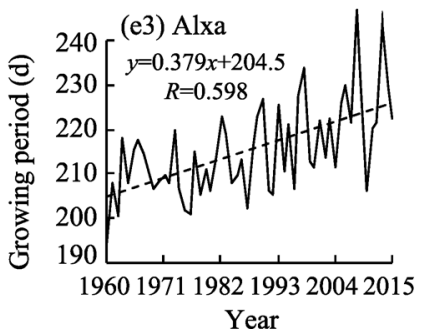

(c1) Qaidam $-0.138 x+102.23$

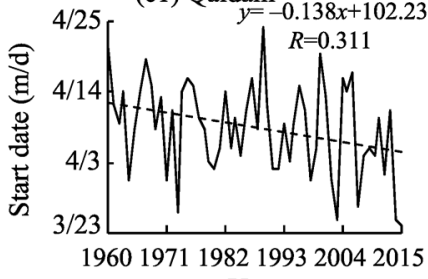

Year

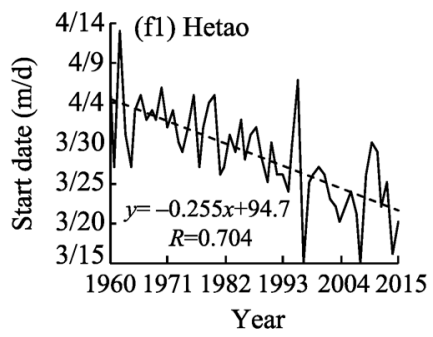

(c2) Qaidam

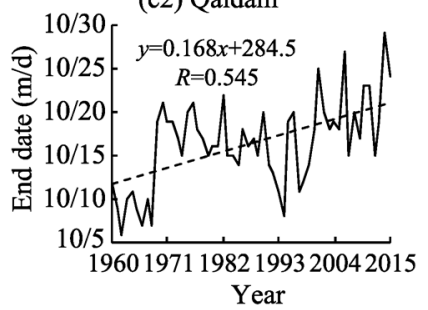

(f2) Hetao

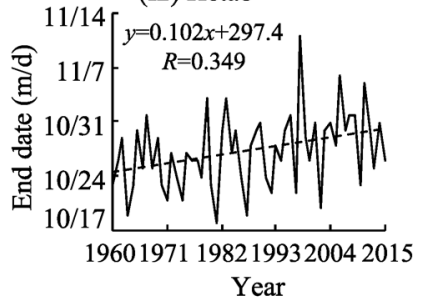

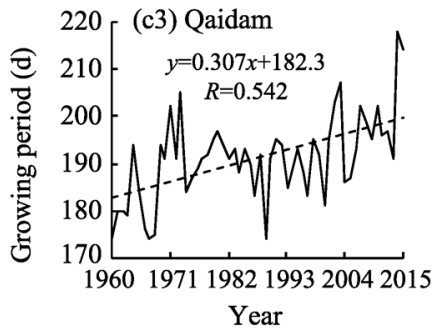

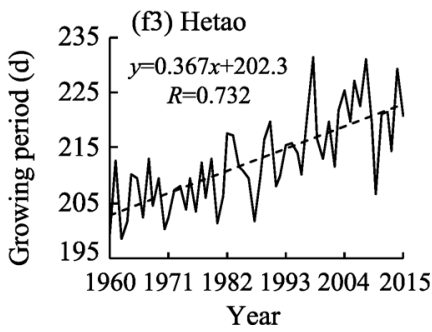

Figure 3 Trends in P. euphratica start date, end date, and growing season variation in each of the Chinese oases sampled in this study (Abbreviations: Beijiang, northern Xinjiang oasis; Nanjiang, southern Xinjiang oasis; Hexi, Hexi Corridor oasis; Hetao, Hetao Plain oasis; Qaidam, Qaidam Basin oasis; Alxa, Alxa oasis) 
positive over this period, from 0.5 to 3.6 , while the greatest change was seen between the 1990 s and the 2000s. This anomaly reached its highest level of 3.6 days during the 2000s, which shows that the most significant trend towards growing season postponement was at this time.

(3) The number of growing season days

Results show that over the past 56 years, the number of days that comprise the growing season of $P$. euphratica in Chinese oases has tended to extend at a rate of $2.66 \mathrm{~d} / 10$ a ( $\alpha \geqslant$ 0.001 ) (Figure 2c). In total, the number of days that comprise the growing season has been extended by 15 days over the past 56 years. Data show that the average growing season for $P$. euphratica is 221 days overall; the longest recorded period was 235 days in 2008 while the shortest was 209 days in 1960. The number of days that comprise the growing season in each oasis are different but have nevertheless all been extended to varying degrees (Figure 3a3-3f3); an average number of 234 days comprise the $P$. euphratica growing season in the southern Xinjiang oasis, the longest across the whole area, while that of the Qaidam Basin oasis was the shortest at just 191 days. The most obvious lengthening trend was seen in the Alxa Plateau oasis, a rate of $3.79 \mathrm{~d} / 10 \mathrm{a}(\alpha \geqslant 0.001)$; in this case, the growing season has been extended by 21 days over the last 56 years. The least obvious lengthening trend was seen in the southern Xinjiang oasis, a rate of change of just $2.11 \mathrm{~d} / 10 \mathrm{a}(\alpha \geqslant 0.001)$ which corresponds to an extension of just 11.8 days over the last 56 years.

In terms of inter-decadal variations (Table 1), the results of this study show that the growing season of $P$. euphratica has been remarkably extended. Negative values characterize the 1960 s to the 1980 s while absolute anomaly values decrease gradually, and the number of days that comprise the growing season exhibit a trend towards significant extension. In the 1990s, however, anomaly values changed from negative to positive, and increased gradually, so the largest changes were seen during the period between the 1960 s and the 2000s; over this time, values increased to 6.6 days and reached a maximum value of 7.3 days by the 2000s. These data reveal an obvious trend towards lengthening the growing season.

In sum, the $P$. euphratica growing season in Chinese oases conforms to a trend over the last 56 years such that the start date has been advanced, the end date has been delayed, and the number of days that comprise this period have been extended. This result is consistent with the conclusion that the spring phenology of woody plants has been advanced by increasing temperatures, while autumn phenology has been delayed, and the number of days that comprise the growing season has increased (Zhang, 1995). Thus, a trend in $P$. euphratica growing season start date advancement is more obvious than the corresponding postponement trend in end date and is also consistent with the conclusion that extension of the growing season is mainly the result of spring phenology advancement (Chmielewski and Rötzer, 2001).

Table 1 Decadal $P$. euphratica growing season anomalies in Chinese oases

\begin{tabular}{cccc}
\hline Decade & Start date $(\mathrm{d})$ & End date $(\mathrm{d})$ & Growing season $(\mathrm{d})$ \\
\hline $1960-1969$ & 1.8 & -2.7 & -4.4 \\
$1970-1979$ & 2.4 & -1.3 & -3.7 \\
$1980-1989$ & 1.6 & -1.5 & -3.2 \\
$1990-1999$ & -0.1 & 0.5 & 0.7 \\
$2000-2009$ & -3.8 & 3.6 & 7.3 \\
$2010-2015$ & -3.3 & 2.3 & 5.5 \\
\hline
\end{tabular}




\subsection{Spatial variations in the $P$. euphratica growing season in Chinese oases}

We investigated the spatial distribution characteristics of mean start and end date values as well as the number of growing season days measured at 48 meteorological stations within Chinese oases between 1960 and 2015 by applying the IDW interpolation method (Figure 4).
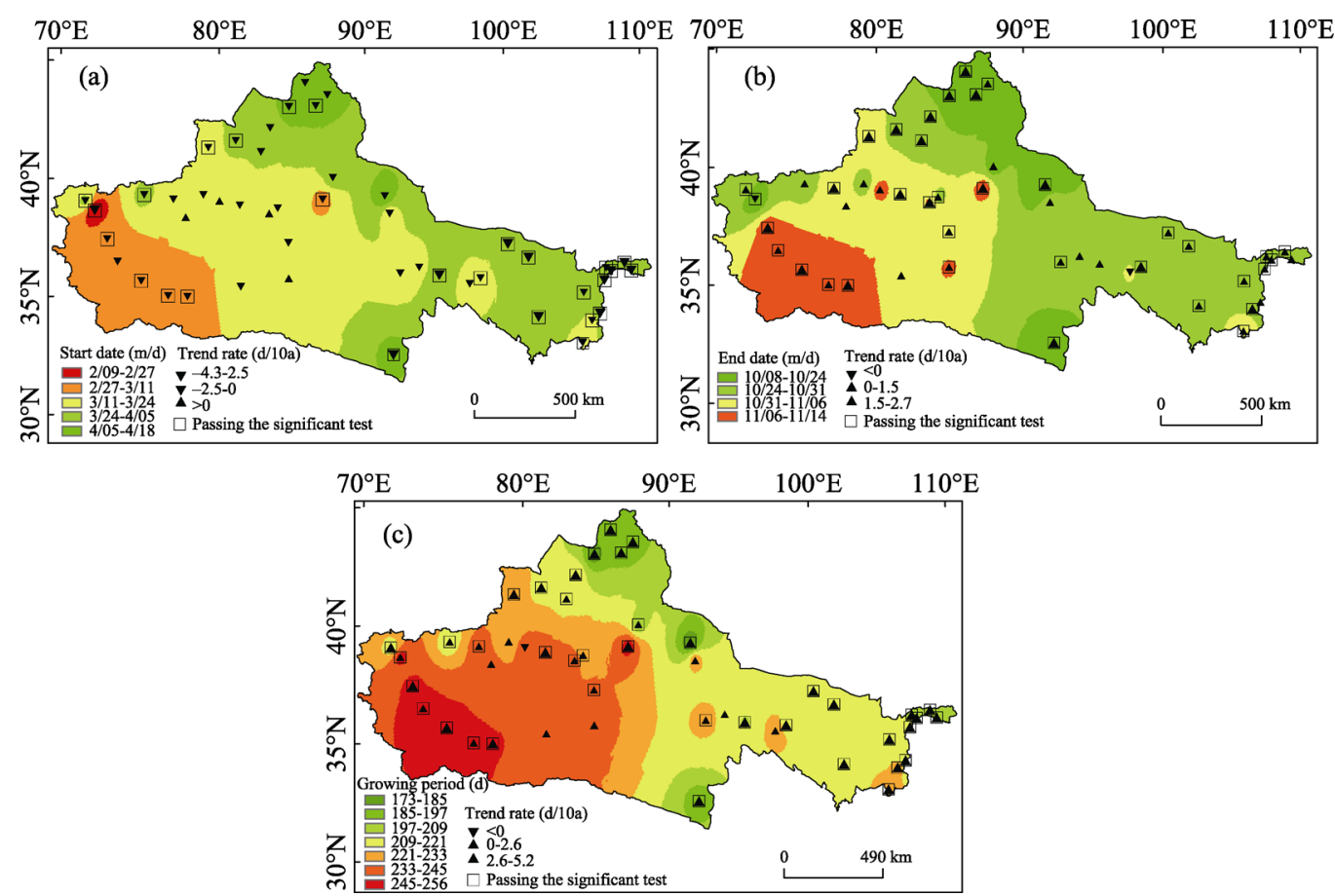

Figure 4 Trends in the spatial distribution of the $P$. euphratica growing season in the Chinese oases sampled in this study

The results of these comparisons show that although spatial disparity in start and end dates, as well as the number of days that comprise the $P$. euphratica growth period exhibit significant regional differences, the characteristics of spatial variation remain consistent. In general, the number of days that make up the growth period have tended to shorten over time; specifically, start dates have tended to be delayed while end dates have advanced from southwest to northeast over Chinese oases. This variation is particularly evident in the Xinjiang oasis; overall, the start date of the growing season has varied between February 9th and April 18th, the end date has varied between October 8th and November 14th, and the number of days that comprise this period has ranged between 173 and 256 . The earliest growing season start date, the latest end date, and the longest growing season were all seen in southwestern China in the southern Xinjiang oasis, for two reasons. In the first place, this oasis is located in the Tarim Basin and is therefore blocked by the Tianshan Mountains; this terrain occlusion means that more cold air enters from the east but is greatly weakened when it reaches the southwest of this oasis $(\mathrm{Li}, 1991)$. Secondly, the underlying surface of this region comprises desert and so it is difficult for heat to spread due to basin topography. This means that the region to the west of southern Xinjiang oasis tends to be warmer than other areas. Similarly, due to the combination of high elevation and low temperature, the Qaidam Basin oasis exhibited the latest start date, the earliest end date, and the shortest growing season. 
Results from $92 \%$ of the stations across our study area reveal a tendency towards advancing start dates for the $P$. euphratica growing season, and $56 \%$ were significant at the 0.05 level (Figure $4 \mathrm{a}$ ). In terms of end dates, delaying trends were seen at about $96 \%$ of stations, and $75 \%$ were significant at the 0.05 level (Figure $4 \mathrm{~b}$ ). In contrast, in terms of the number of days that comprise the growing season, extending trends were seen in about $98 \%$ of stations, and $85 \%$ were significant at the 0.05 level (Figure $4 \mathrm{c}$ ). These data reveal a very obvious and widespread trend towards extending the number of days that comprise the $P$. euphratica growing season in Chinese oases. At the same time, however, start dates were advanced, the number of days that comprised the growing season increased, and end dates revealed an insignificant trend towards delaying within the Hetao and Alxa oases. These observations may be due to the fact that the most significant warming trends occur in winter and summer, while this trend is less significant in spring, and negligible in autumn in Inner Mongolia (Chen et al., 2009). Similarly, an advancing trend in start date combined with a delayed trend in end date, and an extension in the number of days that comprise the $P$. euphratica growing season in southern Xinjiang were the least significant across the study area. In contrast, in the northern Xinjiang region, the end date of the growing season exhibited the most significant trend towards a delay, while the start date exhibited an insignificant advance across this region, a result in close agreement with the earlier work of Li et al. (2011). These workers note that the seasonal warming rate in spring was the lowest in Xinjiang Province between 1961 and 2005. Results also show that the end date of the growing season was significantly delayed within the Qaidam Basin oasis, in concert with an insignificant trend in advancing start date that is related to the autumn and winter warming rate; previous work has shown that this rate is significantly higher than that of the spring and summer, and that spring within this basin is characterized by the slowest rate of warming (Shi et al., 2005).

\subsection{Mutation analysis}

We performed a mutation test analysis to investigate trends in start and end dates as well as the number of days that comprise the $P$. euphratica growing season in Chinese oases over the last 56 years by applying the Mann-Kendall method with a 0.01 significance level and a critical line set at $\mathrm{U}_{\alpha}= \pm 2.56$. UF is the statistic sequence which is calculated by time series sequence. And $\left|\mathrm{UF}_{\mathrm{i}}\right|>\mathrm{U}_{\alpha}$ indicates that the sequence has obvious changing trend.

The results of this analysis show that, out of the last 56 years, a mutation in growing season start date occurred in 2001 (Figure 5a); in other words, the start date of the growing season advanced after 2001, and the UF curve has exceeded the critical line since 2005 when the start date advanced significantly. In contrast, prior to 2001, the average start date of the growing season was March 22nd, and it was March 17th after 2001, this has advanced 5 days following mutation (Table 2). A similar mutation in the end date of the growing season
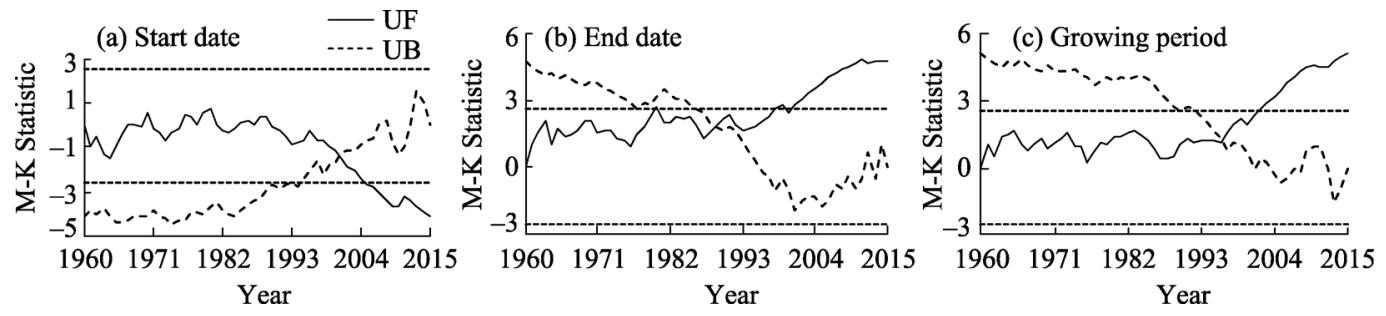

Figure 5 Mutation analysis of the start date, end date, and growing season of $P$. euphratica in Chinese oases 
occurred in 1989 (Figure 5b); results show that the UF curve has exceeded the critical line since 2000 and thus the end date of the growing season has been significantly delayed; the average end date before mutation in 1989 was October 26th, but has subsequently moved to Oc-
Table 2 Changes in the $P$. euphratica growing season in Chinese oases before, and after mutation

\begin{tabular}{lccc}
\hline & Start date & End date & Growing season $(\mathrm{d})$ \\
\hline Before abrupt change & $3 / 22$ & $10 / 26$ & 218 \\
After abrupt change & $3 / 17$ & $10 / 30$ & 228 \\
Change (d) & -5 & 4 & 10 \\
\hline
\end{tabular}
tober 30th, a delay of 4 days. Finally, a mutation in the number of days that comprise the growing season occurred in 1996 (Figure 5c); UF curves indicate that the number of days comprising the $P$. euphratica growing season had been slowly extending prior to 1996, but that a very significant extension has occurred since this date. The average length of the growing season was 218 days before the 1996 mutation, rising to 228 days afterwards, a total extension of 10 days. Thus, the end date of the P. euphratica growing season appears to have exhibited the most sensitive response to climate warming.

\subsection{Period analysis}

We utilized Morlet wavelet power spectra in order to analyze cyclical variations in the start and end date, as well as the number of days that comprise the growing season of $P$. euphratica in Chinese oases. The results of this analysis reveal that the periods of $P$. euphratica growing seasons across the study area were obviously short; amongst these, start dates are characterized by periods of 3.56 years and 4.28 years $(\alpha \geqslant 0.1)$ (Figure 6a; PSD is power spectral density), while end date are characterized by periods of 5.31 years, 5.85 years, and 7.14 years $(\alpha \geqslant 0.1)$ (Figure $6 b$ ), and the number of days within the growing season had a period of 7.09 years $(\alpha \geqslant 0.15)$ cycle (Figure $6 \mathrm{c}$ ). These $P$. euphratica growing season periods are all consistent with an El Niño period of between two years and seven years, while the growing season start date period is consistent with an atmospheric circulation period of between two years and four years. These data are clearly consistent with the fact that the start date, end date, and length of the P. euphratica growing season have mainly been impacted by El Niño events and atmospheric circulation.
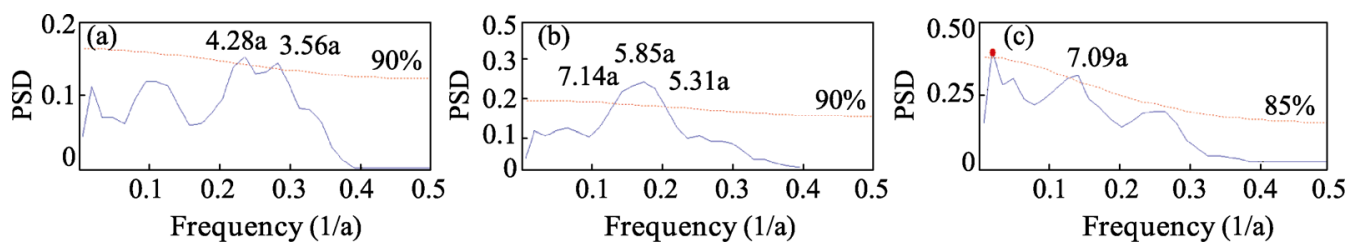

Figure 6 Morlet wavelet power spectra of the start date, end date, and the number of days that comprise the $P$. euphratica growing season in Chinese oases

\subsection{Causal analysis}

(1) Correlation analysis to determine the factors influencing variation

In order to explore the factors that influence changes in the start date, end date, and the number of days that comprise the $P$. euphratica growing season in Chinese oases, we evaluated six circulation indexes as natural proxies (i.e., APVAI, APVII, CA, TPI, and WCI) as well as the artificial CDE. We performed a linear correlation analysis to calculate the corre- 
lation coefficient between atmospheric circulation factors and the start date, the end date, and the number of days that comprise the $P$. euphratica growing season in Chinese oases in corresponding months and periods. We also established a correlation between start dates, end dates, and the number of growing season days in the case of the Qaidam Basin oasis as well as mean values for each index for April, November, and the period between April and November.

The results of this analysis indicate that changes in the start date, the end date, and the number of days that comprise the $P$. euphratica growing season were influenced mainly by the atmospheric circulation factors, such as APVAI, TPI and WCI, and the artificial CDE indexes (Table 3), a finding that is consistent with the periodic characteristics of Morlet wavelet power spectra which proves that the $P$. euphratica growing season have mainly been impacted by atmospheric circulation. Thus, as the APVAI increased, start dates were delayed, end dates advanced, and the number of days that comprised the growing season were extended accordingly. In addition, results also show that the TPI is significantly correlated with start dates, end dates, and the number of days that comprise the P. euphratica growing season, especially within the Hexi Corridor and southern Xinjiang oasis in large part due to the dynamic and thermal effects of the Tibetan Plateau. Indeed, because of the branching effect of the Tibetan Plateau in winter, one cold air branch from the east moves off into southern Xinjiang Province, while the other moves along the Hexi Corridor to the south and consequently influences both the start and end dates of the $P$. euphratica growing season (Wang, 2007). In addition, with the exception of the Qaidam Basin oasis, values for the WCI were

Table 3 Correlation analysis results comparing the $P$. euphratica growing season and relevant impact factors in Chinese oases

\begin{tabular}{|c|c|c|c|c|c|c|c|}
\hline & & APVII & APVAI & $\mathrm{CA}$ & TPI & WCI & CDE \\
\hline \multirow[t]{6}{*}{ Start date } & Alxa & $0.243^{*}$ & $0.529^{* * *}$ & 0.029 & -0.185 & $-0.399^{* * *}$ & $-0.377^{* * *}$ \\
\hline & Beijiang & $0.393^{* * *}$ & $0.583^{* * *}$ & -0.053 & -0.177 & $-0.263^{* *}$ & $-0.246^{*}$ \\
\hline & Qaidam & -0.091 & 0.013 & -0.108 & $-0.346^{* * *}$ & -0.092 & -0.17 \\
\hline & Hetao & 0.173 & $0.486^{* * *}$ & 0.045 & $-0.306^{* *}$ & $-0.397^{* * *}$ & $-0.582^{* * *}$ \\
\hline & Hexi & 0.181 & $0.580^{* * *}$ & -0.016 & $-0.434^{* * *}$ & $-0.409^{* * *}$ & $-0.319^{* *}$ \\
\hline & Nanjiang & -0.21 & $0.342^{* * *}$ & $-0.272^{* *}$ & $-0.540^{* * *}$ & $-0.435^{* * *}$ & $-0.321^{* *}$ \\
\hline \multirow[t]{6}{*}{ End date } & Alxa & -0.104 & $-0.273^{* *}$ & -0.163 & $0.372^{* * *}$ & $0.315^{* *}$ & $0.281^{* *}$ \\
\hline & Beijiang & $-0.324^{* *}$ & $-0.523^{* * *}$ & -0.179 & $0.372^{* * *}$ & $0.284^{* *}$ & $0.547^{* * *}$ \\
\hline & Qaidam & $-0.340^{* *}$ & -0.042 & 0.071 & 0.135 & 0.068 & $0.474^{* * *}$ \\
\hline & Hetao & 0.145 & -0.211 & -0.096 & $0.395^{* * *}$ & $0.382^{* * *}$ & $0.392^{* * *}$ \\
\hline & Hexi & -0.03 & $-0.395^{* * *}$ & -0.122 & $0.581^{* * *}$ & $0.308^{* *}$ & $0.406^{* * *}$ \\
\hline & Nanjiang & $-0.230^{*}$ & $-0.416^{* * *}$ & -0.012 & $0.481^{* * *}$ & $0.236^{*}$ & $0.529^{* * *}$ \\
\hline \multirow{6}{*}{$\begin{array}{l}\text { Growing } \\
\text { season }\end{array}$} & Alxa & -0.152 & $-0.358^{* * *}$ & $-0.246^{*}$ & $0.301^{* *}$ & $0.356^{* * *}$ & $0.507^{* * *}$ \\
\hline & Beijiang & -0.205 & $-0.368^{* * *}$ & -0.152 & $0.345^{* * *}$ & $0.234^{*}$ & $0.538^{* * *}$ \\
\hline & Qaidam & $-0.393^{* * *}$ & $-0.270^{* *}$ & -0.062 & 0.192 & 0.059 & $0.423^{* * *}$ \\
\hline & Hetao & -0.106 & $-0.363^{* * *}$ & -0.22 & $0.361^{* * *}$ & $0.414^{* * *}$ & $0.649^{* * *}$ \\
\hline & Hexi & -0.169 & $-0.362^{* * *}$ & -0.197 & $0.445^{* * *}$ & $0.335^{* *}$ & $0.488^{* * *}$ \\
\hline & Nanjiang & $-0.222^{*}$ & $-0.480^{* * *}$ & 0.041 & $0.592^{* * *}$ & $0.322^{* *}$ & $0.552^{* * *}$ \\
\hline
\end{tabular}

Abbreviations: $* * *$ and, $* * *$ denote $\alpha=0.1, \alpha=0.05$, and $\alpha=0.01$ levels of significance, respectively. 
significantly correlated with the start date, the end date, and the number of days that comprise the $P$. euphratica growing season in other Chinese oases because these areas are all located at the level of mid-latitude westerlies and therefore the intensity of circulation affects temperature. In addition, and again with the exception of the start date in the Qaidam Basin oasis, start dates, end dates, and the number of days that comprise the growing season in other oases are all significantly correlated with $\mathrm{CDE}$; excessive $\mathrm{CO}_{2}$ emissions are the main cause of global warming, and therefore a high degree of correlation between this index and $P$. euphratica growing season variations further reflects the fact that these changes are very sensitive to climatic fluctuations in Chinese oases.

(2) The influence of geographical parameters

As the Chinese oases evaluated in this study cross 14 latitudes from south-to-north and encompass large changes in terrain slope, we further analyzed the influence of latitude and altitude on aspects of the $P$. euphratica growing season. We therefore established a binary linear regression model in this study that expresses the relationship between start date, end date, the number of days that comprise the growing season, and latitude and altitude (see equations (1) to (3), below). Thus, at a 95\% confidence level, corresponding $\mathrm{R}^{2}$ values are $0.44,0.55$, and 0.57 respectively, while $\mathrm{F}$ values are 17,27 , and 29 . Therefore, we obtain the following equations:

$$
\begin{aligned}
& Y_{1}=3.69 X_{1}+0.016 X_{2}-89.4 \\
& Y_{2}=-2.57 X_{1}-0.012 X_{2}+420 \\
& Y_{3}=-6.30 X_{1}-0.028 X_{2}+511
\end{aligned}
$$

where $Y_{1}, Y_{2}$, and $Y_{3}$ denote the start date, the end date, and the number of days that comprise the $P$. euphratica growing season, respectively, while $X_{1}$ denotes latitude, and $X_{2}$ denotes altitude.

This model reflects the effect of both latitude and altitude on the start date, the end date, and the number of days that comprise the $P$. euphratica growing season. Thus, given an increase in latitude of $1^{\circ} \mathrm{N}$, the growing season start date will occur about 3.69 days later, while the end date will occur about 2.57 days earlier, and the number of days that make up the growing season will be shortened by about 6.3 days. Similarly, given a change in altitude of $100 \mathrm{~m}$, the start date will occur about 1.6 days later, the end date will occur about 1.2 days earlier, and the number of days that comprise the growing season will be shortened by 2.8 days. At the same altitude, therefore, a higher latitude will be characterized by a later starting date, an earlier ending date, and a shorter $P$. euphratica growing season, while the same latitude but higher altitude will exhibit a later starting date, an earlier ending date, and a shorter growing season. This analysis shows that the influence of latitude on growing season variables is more significant than that of altitude, while the start date is more significantly influenced by both latitude and altitude than the end date. This result is also consistent with the conclusion that inter-annual variability has more significantly advanced the start date of the growing season as opposed to postponing the end date.

(3) The relationship between the $P$. euphratica growing season and monthly mean temperature

We performed a further correlation analysis as part of this study to investigate the relationship between start date, end date, the number of days that comprise the growing season, and the average temperature of March, October, and between March and October. We also sought to explore response mechanisms between $P$. euphratica growing season variation and 
temperature (Figure 7).

The results of this analysis reveal that the start date of the $P$. euphratica growing season in Chinese oases and March average temperatures are significantly negatively correlated with one another (correlation coefficient: $-0.875 ; \alpha \geqslant 0.001)$. The southern Xinjiang oasis exhibits the highest correlation within this dataset (correlation coefficient: $-0.920 ; \alpha \geqslant 0.001$ ), while the Qaidam Basin oasis has the lowest correlation (correlation coefficient: $-0.265 ; \alpha \geqslant 0.05$ ). At the same

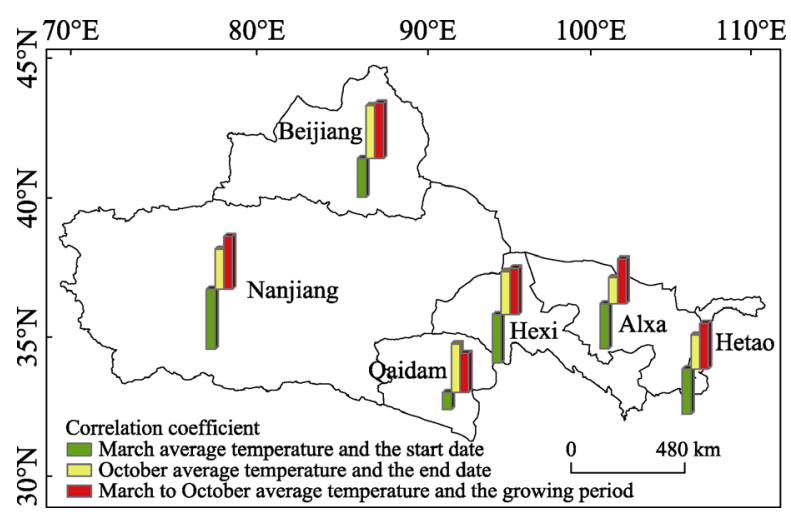

Figure 7 Correlation analyses comparing start dates, end dates, and the number of days that comprise the $P$. euphratica growing season versus mean monthly temperatures in Chinese oases time, data show that the correlation coefficient between the start date of the P. euphratica growing season in the Qaidam Basin oasis and April average temperature was $-0.571(\alpha \geqslant 0.001)$, a result that is clearly related to the high elevation of this region. Similarly, the end date of the P. euphratica growing season in Chinese oases and average October temperature were significantly positively correlated with one another (correlation coefficient: $0.770 ; \alpha \geqslant 0.001$ ), while the end date in each oasis was also significantly correlated with October average temperature. This result shows that the higher the temperature in October, the later the growing season of $P$. euphratica will end; indeed, amongst these oases, the Northern Xinjiang example exhibited the highest correlation (correlation coefficient: $0.815 ; \alpha \geqslant 0.001$ ), while the number of days comprising the $P$. euphratica growing season and the average temperature between March and October were also significantly positively correlated (correlation coefficient: $0.897 ; \alpha \geqslant$ 0.001). In addition, the number of days that comprised the growing season in each oasis were also significantly positively correlated with the average temperature between March and October (correlation coefficients between 0.606 and $0.854 ; \alpha \geqslant 0.001$ ), with the Northern Xinjiang example exhibiting the highest correlation. These data indicate that the higher the temperature between March and October, the longer the P. euphratica growing season.

Previous research has shown that temperature is the dominant meteorological factor that influences phenological changes in woody plants across China (Zhang, 1995). This variable can control phenological arrival time; indeed, the statistical analysis presented above shows that the annual $P$. euphratica growing season is strongly correlated with average temperature in any given month. We therefore established another series of regression equations that express the relationships between start date, end date, the number of days that comprise the growing season, and the average temperature of each corresponding month. Thus, at a $95 \%$ confidence level, corresponding $\mathrm{R}^{2}$ values are $0.77,0.59$, and 0.81 respectively, while $\mathrm{F}$ values are 177,78 , and 223 . Therefore, we obtain the following equations:

$$
\begin{aligned}
& Y_{1}=-2.21 X_{3}+84.78 \\
& Y_{2}=2.76 X_{4}+280.58 \\
& Y_{3}=7.78 X_{5}+116.64
\end{aligned}
$$

where $Y_{1}, Y_{2}$, and $Y_{3}$ denote the start date, the end date, and the number of days that comprise 
the $P$. euphratica growing season, respectively, while $X_{3}$ is the average temperature in March, $X_{4}$ is the average temperature in October, and $X_{5}$ is the average temperature between March and October.

The model accurately reflects the responses seen in the $P$. euphratica growing season to changes in temperature. Calculations show that if the average temperature in March increases by $1{ }^{\circ} \mathrm{C}$, then the growing season will start about 2.21 days earlier, while if the average temperature in October increases by $1{ }^{\circ} \mathrm{C}$, then the end date of the growing season will be delayed by 2.76 days. These results are similar to those reported previously by Chen and $\mathrm{Xu}$ (2012); these workers noted that if the average temperature in the autumn increased by $1{ }^{\circ} \mathrm{C}$, then the end date of the vegetation growing season in the Chinese temperate zone would be delayed by 2.6 days (Chen and $\mathrm{Xu}, 2012$ ). Similarly, if the average temperature between March and October increased by $1{ }^{\circ} \mathrm{C}$, then the number of days that comprise the growing season will be prolonged by about 7.78. These results further indicate that changes in the $P$. euphratica growing season are sensitive in response to global warming.

\section{Conclusions}

(1) The data presented in this study show that the start date of the P. euphratica growing season has advanced over the last 56 years, while the end date has been postponed, and the number of days that comprise the growing season have been gradually prolonged, at average rates of $-1.34 \mathrm{~d} / 10 \mathrm{a}, 1.33 \mathrm{~d} / 10 \mathrm{a}$, and $2.66 \mathrm{~d} / 10 \mathrm{a}(\alpha \geqslant 0.001)$, respectively. Data show that the start date of the $P$. euphratica growing season in the southern Xinjiang oasis was the earliest, while the end date in this region was the latest, and the number of days that comprise the growing season was the longest. This situation is reversed in the case of the Qaidam Basin oasis.

(2) The spatial distribution of start dates, end dates, and the number of days that comprise the $P$. euphratica growing season exhibit significant regional differences, but consistent patterns of spatial variation. The data presented in this paper generally show that the number of days that comprise the growing season have tended to shorten over time, while start dates have been delayed, and end dates have advanced along a transect from southwest-to-northeast over Chinese oases. These patterns of variation are most notable within the Xinjiang oasis.

(3) The mutation analysis presented in this study shows that mutation points of start and end dates as well as the overall growing season were seen in 2001, 1989, and 1996, respectively. Results reveal that the end date of the growing season provides the most sensitive response to climate warming, while analysis of Morlet wavelet power spectra show that the start date, the end date, and the number of days that comprise the $P$. euphratica growing season have cycled over short periods of time between 3.56 years and 7.14 years. This periodicity is consistent with El Niño event cycles, while variation in start dates are likely correlated with atmospheric circulation.

(4) The causal analysis presented in this paper shows that the APVAI, APVII, CA, and CDE indexes provide the main explanations for variations in start dates, end dates, and the number of days that comprise the $P$. euphratica growing season in Chinese oases. This result is consistent with the previous outcomes of Morlet wavelet power spectra analysis. 
Data show that given a $1^{\circ} \mathrm{N}$ increase in latitude, the start date of the growing season will occur about 3.69 days later, the end date will occur about 2.57 days earlier, and the number of days that comprise the growing season will be shortened by about 6.3. Similarly, at an altitude of $100 \mathrm{~m}$, the start date of the growing season will occur about 1.6 days later, while the end date will occur about 1.2 days earlier, and the number of days that comprise the growing season will be shortened by about 2.8. Latitude exerts a more significant influence on the $P$. euphratica growing season than altitude, while the start date was more significantly perturbed by latitude and altitude than the end date.

Data show that the start date, the end date, and the number of days that comprise the growing season were all significantly correlated with the average temperatures in March, October, and between March and October, respectively, with correlation coefficients of $-0.875,0.770$, and $0.897(\alpha \geqslant 0.001)$, respectively. Thus, a $1{ }^{\circ} \mathrm{C}$ increase in March average temperature will cause the start date of the growing season to occur about 2.21 days earlier, while a similar increase in October average temperature will cause a 2.76 day delay to the growing season end date. Lastly, a $1{ }^{\circ} \mathrm{C}$ increase in the average temperature between March and October will cause a 7.78 day increase in the length of the P. euphratica growing season.

\section{References}

Bai J, Ge Q S, Dai J H, 2009. Response of woody plant phenophases to climate change for recent 30 years in Guiyang. Geographical Research, 28(6): 1606-1614. (in Chinese)

Beaubien E G, Freeland H J, 2000. Spring phenology trends in Alberta, Canada: Links to ocean temperature. International Journal of Biometeorology, 44(2): 53-59.

Chang Z F, Qiu G Y, Zhao M et al., 2009. Responses of plant phenology to climate warming in Minqin desert area. Acta Ecologica Sinica, 29(10): 5195-5206. (in Chinese)

Chen X Q, Pang C, Xu L et al., 2015. Spatiotemporal response of Salix matsudana's phenophases to climate change in China's temperate zone. Acta Ecologica Sinica, 35(11): 3625-3635. (in Chinese)

Chen X Q, Peng J D, Li H M, 2009. Seasonal and regional differences of air temperature changes in Inner Mongolia. Geographical Research, 28(1): 27-35. (in Chinese)

Chen X Q, Xu L, 2012. Temperature controls on the spatial pattern of tree phenology in China's temperate zone. Agricultural and Forest Meteorology, 154/155: 195-202.

Chen X Q, Yu R, 2007. Spatial and temporal variations of the vegetation growing season in warm-temperate Eastern China during 1982 to 1999. Acta Geographica Sinica, 62(1): 41-51. (in Chinese)

Chmielewski F M, Rötzer T, 2001. Response of tree phenology to climate change across Europe. Agricultural and Forest Meteorology, 108(2): 101-112.

Cola G, Failla O, Maghradze D et al., 2016. Grapevine phenology and climate change in Georgia. International Journal of Biometeorology, 61: 761-773.

Defila C, Clot B, 2001. Phytophenological trends in Switzerland. International Journal of Biometeorology, 45(4): 203-207.

Fang X Q, Yu W H, 2002. Progress in the studies on the phenological responding to global warming. Advances in Earth Sciences, 17(5): 714-719. (in Chinese)

Fitchett J M, Grab S W, Thompson D I et al., 2014. Spatio-temporal variation in phenological response of citrus to climate change in Iran: 1960-2010. Agricultural \& Forest Meteorology, 198-199: 285-293.

Fu Y H, Campioli M, Demarée G et al., 2012. Bayesian calibration of the uUnified budburst model in six temperate tree species. International Journal of Biometeorology, 56: 153-164.

Gao Q, Chen J, Yan F et al., 2012. Phenological characteristics of herbaceous plants in Hebei Province and their responses to climate warming. Chinese Journal of Ecology, 31(3): 600-605. (in Chinese) 
Ge Q S, Dai J H, Zheng J Y, 2010. The progress of phenology studies and challenges to modern phenology research in China. Bulletin of Chinese Academy of Sciences, 25(3): 310-316. (in Chinese)

Juknys R, Kanapickas A, Šveikauskaite I et al., 2016. Response of deciduous trees spring phenology to recent and projected climate change in Central Lithuania. International Journal of Biometeorology, 60(10): 1589-1602.

Li J F, 1991. Xinjiang Climate. Beijing: China Meteorological Press, 75-76. (in Chinese)

Li Q H, Chen Y N, Shen Y J et al., 2011. Spatial and temporal trends of climate change in Xinjiang, China. Journal of Geographical Sciences, 21(6): 1007-1018.

Liu J, Zheng Y F, Zhao G Q et al., 2007. Responses of phenology to climate change in the Zhengzhou area. Acta Ecologica Sinica, 27(4): 1471-1479. (in Chinese)

Liu P X, Zhang K X, 2011. Climate characteristic of seasonal variation and its influence on annual growth period of Populus euphratica populous euphratica Olive in the Hexi Corridor in recent 55 years. Acta Ecologica Sinica, 31(3): 882-888. (in Chinese)

Liu Q, H. Fu Y S, Zhu Z C et al., 2016. Delayed autumn phenology in the Northern Hemisphere is related to change in both climate and spring phenology. Global Change Biology, 22: 3702-3711.

Menzel A, Fabian P, 1999. Growing season extended in Europe. Nature, 397(6721): 659.

Pei S X, Guo Q S, Xin X B et al., 2009. Research on plant phenological responses to climate change abroad. World forestry research, 22(6): 31-37. (in Chinese)

Qin D H, Thomas S et al., 2014. Highlights of the IPCC Working Group I Fifth Assessment Report. Progressus Inquisitiones de Mutatione Climatis, 10(1): 1-6. (in Chinese)

Roetzer T, Wittenzeller M, Haeckel H et al., 2000. Phenology in central Europe: Differences and trends of spring phenophases in urban and rural areas. International Journal of Biometeorology, 44(2): 60-66.

Schwartz M D, Reiter B E, 2000. Changes in North American spring. International Journal of Climatology, 20(8): 929-932.

Shen Y C, Wang J W, Wu G H et al., 2001. Oaseis of China. Zhengzhou: Henan University Press, 278-284. (in Chinese)

Shi X H, Zhao Y N, Dai S et al., 2005. Research on climatic change of Qaidam Basin since 1961. Journal of Desert Research, 25(1): 123-128. (in Chinese)

Sparks T H, Jeffree E P, Jeffree C E, 2000. An examination of the relationship between flowering times and temperature at the national scale using long-term phenological records from the UK. International Journal of Biometeorology, 44(2): 82-87.

Szabó B, Vincze E, Czúcz B, 2016. Flowering phenological changes in relation to climate change in Hungary. International Journal of Biometeorology, 60(9): 1347-1356.

Wang J A, 2007. Chinese Geography Course. Beijing: Higher Education Press, 33-34. (in Chinese)

Wang S J, Chen B H, Li H Q, 1995. Populus Euphratica. Beijing: China Environmental Science Press, 16-18. (in Chinese)

Wei Q J, 1990. Populus Euphratica. Beijing: China Forestry Publishing House, 7-21. (in Chinese)

Zhang F C, 1995. Effects of global warming on plant phonological evertsevents in China. Acta Geographica Sinica, 50(5): 402-410. (in Chinese)

Zhao M L, Liu P X, Zhu X J et al., 2012. Response of Populus euphratica Oliv. Phenology to climate warming in the oasis of lower reaches of Heihe River from 1960 to 2010. Acta Botanica Boreali-Occidentalia Sinica, 32(10): 2108-2115. (in Chinese) 